\title{
Intramurales Duodenalhämatom nach endoskopischer Biopsie
}

Als Komplikation endoskopischer Biopsien bei vermuteter Erkrankung des Duodenums stellen intramurale Duodenalhämatome (IDH) eine Rarität dar. Am häufigsten treten IDH nach einem stumpfen Bauchtrauma auf. Auch seltene Fälle als Folge einer Pankreatitis sind in der Literatur belegt. Perforation und Pankreatitis sind als primäre Komplikationen $\mathrm{zu}$ berücksichtigen (Guzman et al., Am J Gastroenterol 1998; 93: 2547). Im folgenden Fallbericht sollen das klinische Bild und die Bilddiagnostik bei einem Patienten mit einem intramuralen Duodenalhämatom diskutiert werden.

\section{Fallbericht}

Bei dem 56-jährigen Patienten waren 10 Tage vor Aufnahme in unsere Klinik im Rahmen einer Endoskopie des oberen Gastrointestinaltraktes wegen einer Duodenitis Probebiopsien entnommen worden. Die stationäre Aufnahme erfolgte wegen zunehmender epigastrischer Schmerzen und Erbrechen. Die körperliche Untersuchung bei Aufnahme erbrachte außer einem Druckschmerz im Oberbauch keinen weiterführenden Befund. Im Labor fielen eine erhöhte GGT (211 U/1) und Lipase (1197 U/1) auf. Sonographisch stellte sich der DHC mit $8 \mathrm{~mm}$ gering dilatiert dar. Die Gallenblase war deutlich vergrößert und sludgegefüllt. Das flüssigkeitsgefüllte Duodenum brach in der Pars descendens an einer glatt begrenzten echoarmen Raumforderung ab. Der Befund zeigte sich in der ÖGD als eine auf Höhe der Papillenregion gelegene Stenosierung des Duodenallumens. Die anschließend durchgeführte kontrastverstärkte CT bestätigte die Sonographie. Es fand sich eine $7 \times 5 \times 4 \mathrm{~cm}$ messende hypodense Raumforderung mit Dichtewerten um $35 \mathrm{HE}$ auf der dem Pankreaskopf abgewandten Seite der Pars descendens des Duodenums in Höhe der Duodenalpapille. Der Befund war gegenüber den distalen Anteilen der ableitenden Gallenwege nicht sicher abgrenzbar. Zur weiteren Differenzierung und Klärung der topographischen Verhältnisse wurde eine kontrastverstärkte MRT einschließlich einer MR-Cholangiopancreatographie (MRCP) durchgeführt. Dabei stellte sich die Raumforderung in $\mathrm{T}_{2}$ - und $\mathrm{T}_{1}$-Gewichtung signalintens dar. Das Pankreas war unauffällig. Die MRCP zeigte einen kompressionsbedingt auf knapp $1 \mathrm{~cm}$ dilatierten DHC und einen betonten D. pancreaticus. Eine direkte Verbindung der Raumforderung zu den ableitenden Gallenwegen konnte ausgeschlossen werden. Die MRT-gestützte Verdachtsdiagnose lautete somit stenosierendes intramurales Duodenalhämatom mit Gallengangsobstruktion. Der Patient wurde zunächst konservativ weiterbehandelt. Wegen anhaltender Oberbauchschmerzen und steigender Cholestase- und Entzündungsparameter sowie persistieren- 


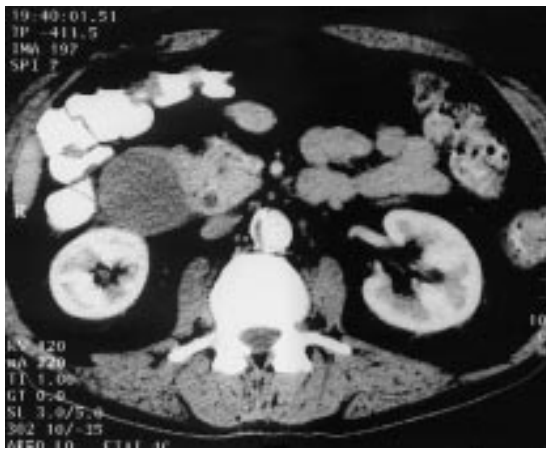

Abb. 1 Kontrastverstärkte CT des Abdomens am 7. Tag nach endoskopischer Biopsie. Intramurales Hämatom in der Pars descendens des Duodenums. Stenosierung des Darmlumens und dilatatierter D. hepatocholedochus im Verlauf durch den Pankreaskopf.
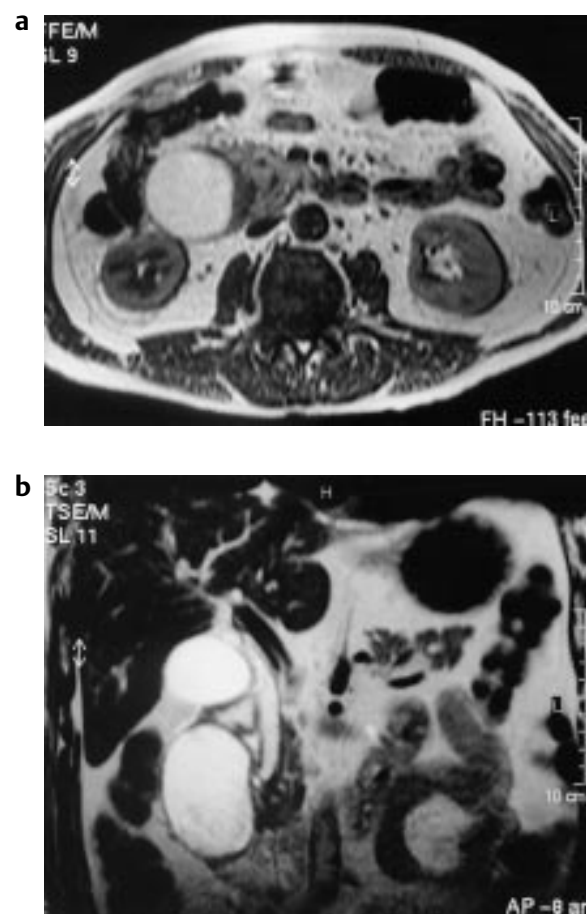

Abb. 2 Kontrastverstärkte axiale (a) und koronare (b) MRT des Oberbauches. In $\mathrm{T}_{2-}$ und $\mathrm{T}_{1}$-Gewichtung hyperintense Raumforderung in der Duodenalwand. Hämatom im Methämoglobinstadium. Dilatierter D. hepatocholedochus. 9. Tag nach endoskopischer Biopsie.

der Darmobstruktion wurde das Hämatom am 12. Tag des Aufenthaltes chirurgisch ausgeräumt. Intraoperativ und histologisch konnte die Verdachtsdiagnose bestätigt werden. Der postoperative Verlauf war problemlos.

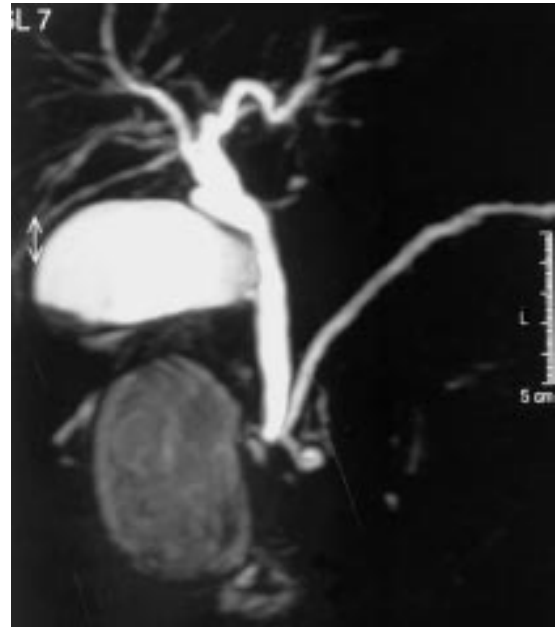

Abb. 3 MR-Cholangiopancreatographie. Räumliche Beziehung des Duodenalhämatoms zur Papilla duodeni major und kompressionsbedingte Dilatation der ableitenden Gallenwege.

\section{Diskussion}

Intramurale Duodenalhämatome als Komplikation endoskopischer Probebiopsien oder endoskopischer Blutstillung sind extrem selten. In großen prospektiven Studien zur Beurteilung des Risikos endoskopischer Eingriffe spielt das IDH zahlenmäßig keine Rolle (Fleischer et al., Gastrointest Endosc 1992; 38: 411). Prädisponierend sind neben medikamentöser Antikoagulation Blutgerinnungs- und Thrombozytenfunktionsstörungen. Auch spontane intramurale Einblutungen kommen bei Blutgerinnungsstörungen vor. In $80 \%$ der Fälle dagegen sind IDH Folge eines stumpfen Bauchtraumas. In dieser Gruppe sind Kinder in der Mehrzahl der Fälle betroffen. Als Ursache wird die schwächere Ausprägung der Bauchmuskulatur diskutiert. Typische Unfallmechanismen sind Dezelerationstraumen und Zweiradlenkerverletzungen. Pathophysiologisch spielen die fixierte Lage des Duodenums und seine topographische Beziehung zur Wirbelsäule, die es Scherkräften beim Trauma bzw. bei einer endoskopischen Probeexzision in hohem Maße aussetzen, und die ausgeprägten submukösen Venenplexus eine wesentliche Rolle (Guzman et al., Am JGastroenterol 1998; 93: 2547). Eine weitere seltene Ursache ist die Pankreatitis, bei der es durch Arrosion duodenaler Blutgefäße durch das Pankreassekret zur Einblutung in die Darmwand kommt. Die klinische Symptomatik ist unspezifisch und umfasst Oberbauchschmerzen,
Abwehrspannung und Erbrechen (Han et al., Hepato-Gastroenterol 1997; 44; 13). Ein Ikterus als Ausdruck der durch das Hämatom bedingten mechanischen Cholestase kann hinzukommen. Als Komplikationen müssen Perforation ins Darmlumen bzw. Mesenterium und mit einer Prävalenz von 6-8\% die akute Pankreatitis berücksichtigt werden. Die Pankreatitis beruht dabei auf einer Obstruktion im Bereich der Papille und dem dadurch bedingten Aufstau des Pankreassekretes. Auch die direkte Kompression und Traumatisierung des Organs durch das Hämatom werden diskutiert. Zeitlich an erster Stelle der bildgebenden Diagnostik steht die Sonographie als schnelle und weithin verfügbare sowie nicht-invasive Untersuchungstechnik. Diagnostische Einschränkungen ergeben sich häufig durch Darmgasüberlagerung und eine begrenzte Ortsauflösung insbesondere bei kleinen Befunden (Han et al., HepatoGastroenterol 1997; 44: 13).

Mit der CT können die Größe und Lage des Hämatoms in der Darmwand und die topographische Beziehung zu Nachbarorganen wie dem Pankreaskopf sicher beurteilt werden. Außerdem können Komplikationen wie Pankreatitis und Perforation, die potentielle Auswirkungen auf das therapeutische Vorgehen haben, erfasst werden. Das computertomographische Erscheinungsbild hängt vom Alter des Hämatoms und der Dichte der umgebenden Parenchymstrukturen ab. Während sich frische Einblutungen überwiegend hyperdens darstellen, nimmt die Dichte mit zunehmendem Alter des Hämatoms ab (Han et al., Hepato-Gastroenterol 1997; 44: 13). Die Strahlenbelastung ist insbesondere bei Kindern zu berücksichtigen. Limitierungen bestehen außerdem in der bildmorphologischen Abgrenzung gegenüber anderen duodenalen Raumforderungen, wenn die Anamnese nicht eindeutig oder ein adäquates Trauma nicht erinnerlich ist.

Das MR-tomographische Erscheinungsbild intramuraler Duodenalhämatome ist nach unserer Kenntnis bislang nicht in der Literatur beschrieben. Die MRT bietet neben ihrer hervorragenden Eignung zur Gewebedifferenzierung auch die Möglichkeit zur exakten Lokalisation der Läsion in der Darmwand (Marcos et al., Magn Reson Imaging 1999; 17: 869). Zudem steht mit der MRCP eine sensitive Methode zur nicht-invasiven Beurtei- 
lung der Gallengänge und des D. pancreaticus zur Verfügung. In unserem Fall konnten die räumliche Beziehung des Hämatoms zur Duodenalpapille und die Aufweitung der Cholangen bzw. des Pankreashauptganges herausgearbeitet werden. In Abhängigkeit vom Stadium des Hämoglobinabbaus und dem Ausmaß der Hämosiderineinlagerung wiesen IDH in der MRT in $\mathrm{T}_{2^{-}}$und $\mathrm{T}_{1}$ Gewichtung variable Signalintensitäten auf (Marcos et al., Magn Reson Imaging 1999; 17: 869). Die morphologische Differenzialdiagnose ist begrenzt und umfasst in erster Linie eingeblutete duodenale Duplikations- und Pankreaspseudozysten.
Das therapeutische Vorgehen ist mit parenteraler Ernährung und nasogastraler Sekretabsaugung zunächst konservativ. Indikationen für eine Operation ergeben sich bei länger als 10 Tage persistierender Obstruktion der Gallenoder Pankreasgänge, bei Perforation oder schwerer kompressionsbedingter Darmschädigung (Sidhu et al., Am J Roentgenol 1998; 170: 38).

M. Sollfrank, W. Koch, H. Waldner, K. Rüdisser, München 